\title{
THE NAME PATHWAY, AN ELEMENTARY COGNITIVE MODEL OF NAMES AS SENSORY REPRESENTATIONS
}

\author{
S. Balachandra Dass \\ Department of Medical Affairs, Regeneron Pharmaceuticals, Inc., Tarrytown, New York ${ }^{1}$
}

Received 2014-03-11; Revised 2014-03-31; Accepted 2014-04-03

\begin{abstract}
Names function as sensory representations, but the relationship between names and sensory stimuli/responses remains unclear. This study proposes the existence of a class of stimulus-response pathways, the name pathway, where a name is a reproducible and communicable symbol and the name pathway is one where the same name is both the stimulus and the response. Once a stimulus-response "name" pathway is formed as a result of reinforcement-based learning and in concert with the formation of the associated stimulus-response "sensory" pathway for a named object, act or process, Hebbian cross-pathway connectivity between the sensory and name pathways allows each pathway's stimulus to activate the other's response. The model proposes that every higher-order cognitive function exists only because it was named and that each such function may be defined mechanistically to be the outcome of the "recognition," "interpretation," and "retrieval" of sensory experiences from networks of names for that function.
\end{abstract}

Keywords: Name Pathway Model, Sensory Experience, Networks, Neural Model, Hebbian Learning

\section{INTRODUCTION}

A number of theories have speculated that the expression of coherent and reproducible language or its elements is an inherent feature of human perception and cognition (Pinker, 1999; Kuhl, 2000; Holden, 2004; Wong, 2005). A large body of empirical and theoretical literature on word acquisition exists (Colunga and Smith, 2005; Regier et al., 2005; Garagnani et al., 2008; Cummings et al., 2009; Frank et al., 2009; Mayor and Plunkett, 2010). Many proposals have advocated a role for symbols and names as sensory representations (Harnad, 1990; Humphreys et al., 1999; Feldman and Narayanan, 2004; Sheridan, 2005). Ideas such as the adaptive resonance theory seek to relate learning and the naming process (Glotin et al., 2010), while other models have implicated dynamic neural states in the naming of actions (Tranel et al., 2008). Several lines of research continue to demonstrate a relationship between names (such as words) and neurons (Quiroga et al., 2005) and between names and perception/cognition (Winawer et al., 2007). Evidence has been presented for networks of brain regions supporting the naming of objects (DeLeon et al., 2007; Newhart et al., 2007).
Nevertheless, these reports remain inadequate in putting forward a self-sufficient neural model of cognition describing a precise and direct structuralfunctional relationship between names and sensory experiences. For instance, there exists a need for a simple and testable proposal of a mechanism that demonstrates, based on the fundamental principles of stimulus-response pathways, how specific sensory experiences may be selectively accessed by names and how sensory experiences can retrieve names. Specifically, what remains lacking is a hypothesis that first postulates the identity and anatomy of that particular neural component responsible for relating names to sensory experiences and then mechanistically outlines such an association.

The theoretical model in this study is a new proposal for the existence of a previously undescribed and unique class of stimulus-response sensory pathways, the name pathway, that describes a direct, one-on-one relationship between names and sensations. It defines, for the first time, this novel pathway as one in which the same name is both the stimulus and the response. Necessary and sufficient mechanisms by which the name pathway serves as a seamless structural

\footnotetext{
${ }^{1}$ Current address. This is the author's own work that was not done at or associated with Regeneron Pharmaceuticals, Inc.
} 
and functional link between names and the sensory experience associated with the named object, act or process are described. The end result of the processes in this pathway is the emergence of uniquely human, higher-order cognition: Comprehensive reasoning and universal understanding, wide-ranging thought and deliberation, evaluable and comparative judgment, semantic and broad-spectrum recall, symbolic manipulation and mathematical computation, cultural communication and transmission-processes that transcend the elementary "consciousness" and rudimentary cognitive traits attributed to non-human animals (Hauser, 2005; Heyes, 2012; Thornton et al., 2012; The CDC, 2012).

Importantly, for such a model to be meaningful, it must be in consonance with one or more current views on the origins of language and cognition and their interrelationship. Along this line, the proposed model is aligned with two overlapping schools of thoughts: The cognitive neuroscience movement that emphasizes neurobiological underpinnings to explain the relationship between language and cognition (Harris, 2003) and the evolutionary cognitive movement that posits natural selection acting on early humans as the force driving the origin of and association between language and cognition (Heyes, 2012; Ulbaek, 1998).

\section{DESCRIPTION OF THE MODEL}

\subsection{Structure of the Name Pathway and its Cross-Connectivity to Sensory Pathways}

A name pathway is defined as a stimulus-response pathway where the same name is both the stimulus and the response. A name is any reproducible and communicable symbol that is learnt, such as an auditory or visual symbol that is a part of language. The development of a name pathway that results from learning (as when the child is able to correctly vocalize the name that he hears of an object shown to him) is associated with another sensory pathway that links the (visual) stimulus of the object to the physical/behavioral response to this stimulus. For convenience, this nonname sensory pathway will be referred to from here on as "sensory pathway," to distinguish it from a name pathway. Because the name pathway is active at the same time as this sensory pathway, both pathways become wired to each other (per Hebb's rule) by a connecting pathway (Fig. 1). The name for an object, act or process has now become linked-or assigned-to the sensory response to the object. In this model, a stimulus activating a sensory pathway connected to a name pathway can result in producing that name (vocal response of saying the name) and a stimulus activating a name pathway connected to a sensory pathway can result in producing the response that is associated with that sensory pathway (such as reaching for the named object). This cross-pathway connectivity and activation between name stimuli and sensory responses and between sensory stimuli and name responses allows names to function as cognitive elements.

Name pathways as defined would include those that connect stimuli of names heard, transcribed or otherwise expressed (such as sign language) to the response of producing the same sound, transcription or expression of the names. The pathways once formed are strengthened and made durable i.e., become established-through constant activation and reinforcement during the learning process and by a variety of mechanisms that could include long-term potentiation and long-term depression (Brecht and Schmitz, 2008).

\subsection{Name Pathway Networks, Prerequisites for Higher-Order Cognitive Activity and the Stimulus-Response Nature of their Wiring}

When the previously established name pathway for the name apple is simultaneously activated with another established name pathway (as when these names are taught together), these pathways become connected. Thus, the apple name becomes connected to names such as see, nice, round, red, smooth, bite, sweet, cut, knife, juicy, take, put, one, on top of, yesterday, small, mine, I, dad and so on, when the apple name is taught in conjunction with or heard together with each of the above names. The result is the creation of an apple name network, consisting of an interconnected network of all the above names, together with their associated sensory pathways. This means that excitation in any one name pathway in a network of established name pathways could activate the name pathways of all the other names; by extension, the activation of any one name pathway in a network now has the potential to activate the responses of all sensory pathways associated with that entire name network.

However, not all pathways in a name network are activated to the same extent, since this is dependent upon the intensity, duration and consistency of activity (Schurger et al., 2010) and the level of reinforcement, together constituting the strength or neural experience of a pathway and its networks. 


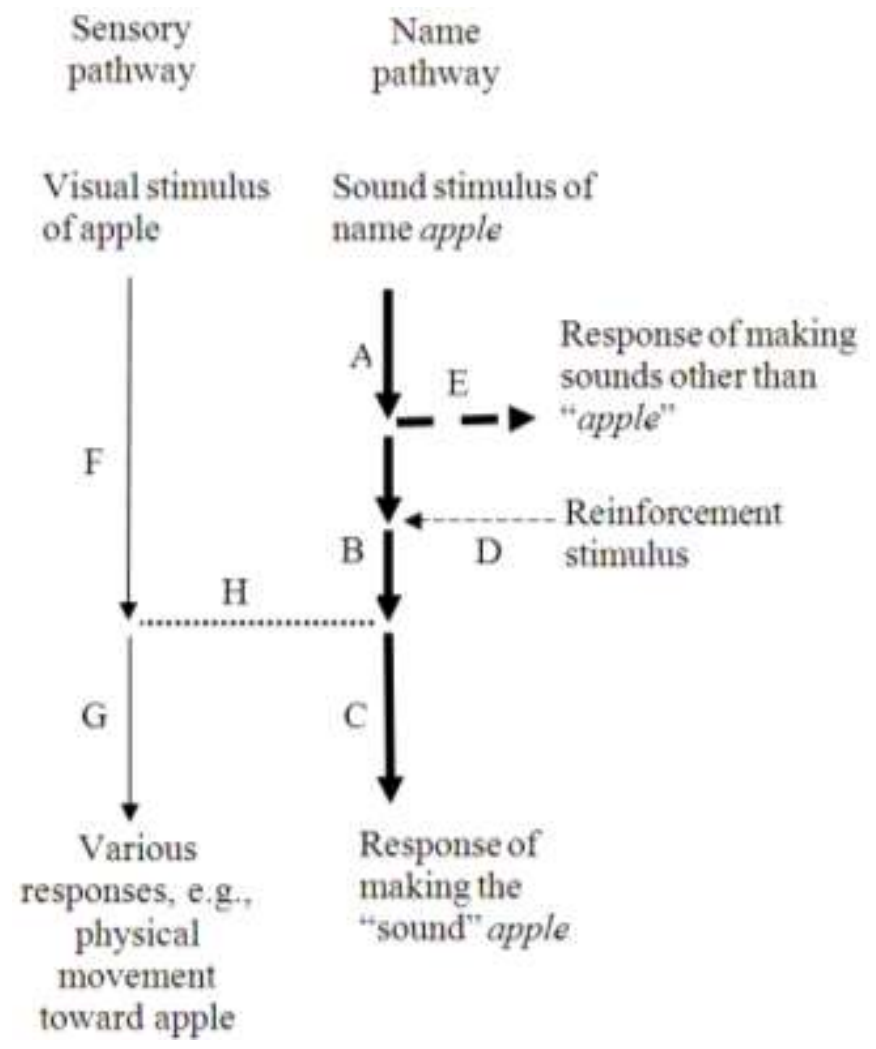

Fig. 1. Formation of a name pathway and its cross-pathway connection to (non-name) sensory pathways. The name pathway in the child for the name apple (thick lines, A, B, C) is formed and strengthened through reinforcement-based learning (reinforcement stimulus from the teaching person, dashed line D), that eventually results in the loss of activity in response pathway E (that produces sounds other than "apple"). Because the (non-name) sensory pathway (thin, unbroken lines, F, G) linking the visual stimulus of the apple with behavioral/physical responses and the name pathway are simultaneously active, they become connected together per Hebb's rule. This connecting pathway H (dotted line) is formed as a bidirectional pathway. The name apple has now become "assigned" to the sensory response to the object apple. Once these pathways are established, subsequent activation of the sensory pathway, as by the visual stimulus of the apple, can bring about a name response in the other pathway via the connecting pathway: F to H to C. Similarly, a name stimulus (such as on hearing the sound of the name) can bring about a response in the sensory pathway: A to B to H to G. Such cross-pathway connectivity and activation is the basis for names to function as cognitive elements, through processes of recognition, interpretation and retrieval

The relative strengths of pathways therefore determine the directions of activity; directional wiring is also the consequence of the sequential activation of name pathways during the learning process. That is, each name is a stimulus to its following name and a response to its preceding name, as when the child learns the name group red apple or on the table after having learned each name separately. This stimulus-response nature of name groups may serve as a basis of the development of syntax and language units such as phrases.

\subsection{Emergence of Higher-Order Cognition via the Name Pathway: Processes of "Recognition," "Interpretation" and "Retrieval" of Sensory Experience}

The establishment of a name pathway and its connection to its sensory pathway permits the subsequent discriminative activation of this sensory pathway via this name. Such categorical excitation of a sensory response as a result of activation of its associated name pathway 
may be described as the identification or "recognition" of sensory experience by a name.

Each name pathway is connected to several sensory pathways. For instance, the see name pathway is connected to visual pathways of a multitude of different objects. Under this condition, differential recognition of only a particular subset of sensory pathways belonging to a name occurs when two or more names are activated in conjunction. Such simultaneous excitation, as when these names are heard together, will cause only those sensory pathways that are shared by both name pathways to be activated the most (Fig. 2). This may be described as the process of "interpretation" of sensory experience, whereby activated names modify/qualify the totality of the sensory responses available to them by recognizing only that subset of sensory pathways common to all their names, thus discriminatively "retrieving" (defined below) a specific subset of experiences.

Processes of activation and deactivation of pathways, possibly functioning in a cyclical manner, appear to be features of perception and cognition (Casey et al., 2000; Haider et al., 2006; Stroffek et al., 2007). Thus, activation of a name or sensory pathway is followed by a deactivation of this pathway that is accompanied by an activation of its connected sensory or name pathway; subsequent deactivation of this secondary sensory or name pathway is accompanied by re-activation of the initial name or sensory pathway. The net effect is a repetitive and continual fraction-of-time by fraction-oftime activation and reactivation of name and sensory pathways in name networks, which may be described as the process of "retrieval" of recognized and interpreted sensory experiences by names.

\subsection{A Name Pathway Model of Higher-Order Cognition}

The fundamental precept of this model is that every higher-order cognitive function exists only because it was named. That is, all higher-order reasoning, deliberation, understanding, judgment, abstract thought, non-episodic recall, computation, communication, etc., that are uniquely characteristic of the human condition may be defined to be the outcome of the recognition, interpretation and retrieval of sensory experiences from networks of names for that function. In this definition of the emergence of higher-order cognition, note the centrality of the five elements of the model that derive from the Hebbian, stimulus-response nature of the name pathway: (1) The initial recognition of sensory experience by names; (2) the subsequent interpretation of such recognized sensory experience by names; (3) the further retrieval of such interpreted sensory experience by names; (4) the overall anatomy of the name network, built over the name pathways, that allows for the retrieval of vast amounts of interconnected sensory experience by just a single name; and (5) the stimulusresponse architecture of name pathways belonging to name groups that may allow for the development of syntax and language units such as phrases.

In the example illustrated in Fig. 3, the sight of a sunset activates visual pathways, setting off a torrent of activity in connected name and sensory pathways in a multitude of overlapping name networks. These would include the network for the name see, consisting of name pathways for see, look, eye, ocean and the network for the name sunset, consisting of pathways for the names sunset, sun, clouds, dusk, red. Because the name see was previously established in concert with the names I and aware in the form of name groups (as defined previously), activity in the see name pathway will now activate the I name network (consisting of pathways for the names $I$, he, $m y, a m, o f)$ and the aware name network (aware, know, understand). An understanding of the visual awareness of the sunset (that transcends the mere object knowledge that an animal may have, as pointed out in section 1) may now be defined as the recognized, interpreted and retrieved sensory experiences of activated networks of all the name pathways listed above (Fig. 3).

These name networks may also excite connected pathways for other names such as pretty or scenic and their networks. The result is a perception of the beauty of the sunset, which may be defined as the recognized, interpreted and retrieved sensory experiences of the networks of names for this perceptive function: Such names would include those above, activated earlier and others activated subsequently, namely, pretty, scenic, grand, attractive, nice, wonder. If there is a sense of well-being on seeing the sunset, this results from the recognized, interpreted and retrieved sensory experiences of activated networks of interconnected names that would now include good, pleasure, calm, feel, happy, lucky and satisfied.

The "thinking" of these names occurs concomitantly, whereby activity in the sensory pathways connected to these name pathways leads to the retrieval of name responses, resulting in my thought of the above names: "This pretty sunset makes me feel happy!" A mechanism for thinking of names is proposed below.

When does a contrary perception of dullness of the sunset become the dominant function? This result follows from the activation of a different network of connected names, such as dull, boring, seen before, yawn, uninteresting (Fig. 3). 


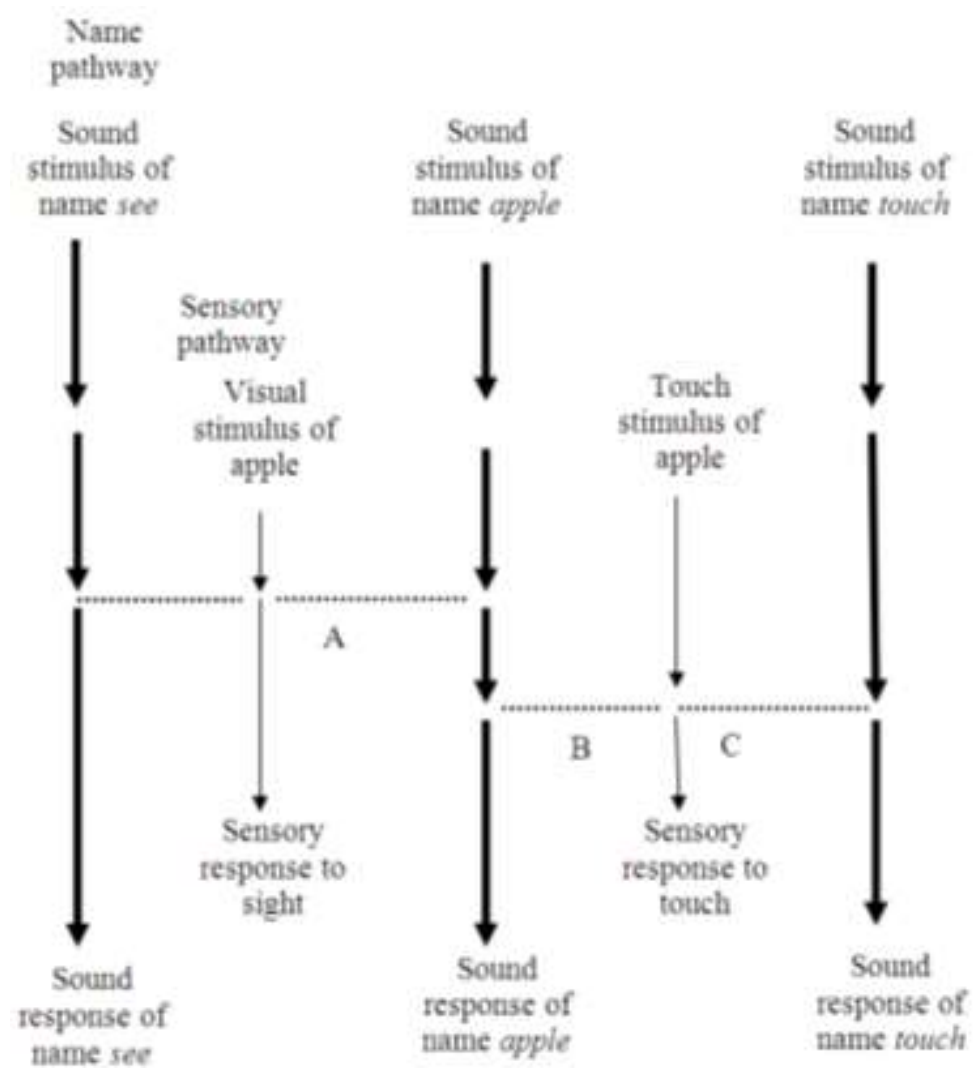

Fig. 2. Interpretation of sensory experiences by names. A limited number of names and sensory pathways are depicted here. As in Fig. 1, name pathways are in thick, vertical lines, while sensory pathways are in thin (unbroken), vertical lines. Interconnections between these pathways are shown in dotted, horizontal lines: A, B, C. On hearing the sound "apple," excitation in the apple name pathway activates the visual and touch sensory pathways equally via connections A and B, respectively. On the other hand, when the apple and touch name pathways are concurrently activated (as when the name group "touch apple" is heard), excitation received by the touch sensory pathway via B and C is stronger than the excitation received by the visual sensory pathway via A alone (two sources of excitation versus one). This results in the net excitation of the touch sensory pathway to the exclusion of activity in the visual sensory pathway. Thus, in this example, although one name (apple) pathway is capable of activating multiple sensory pathways, a second name (touch) modifies or qualifies-that is, interprets-the totality of the sensory responses available to the first name pathway such that only a select subset of sensory responses associated with the first name is actually activated. Such interpretation is part of a continuum of activities that follows the recognition of names and precedes the retrieval of sensory information from networks of name pathways wired in a stimulusresponse, directional manner

For instance, my dull name pathway may be strongly activated because I heard someone say that name as I was watching the sunset or because I saw someone yawn, activating my sensory and name pathways connected to the dull name.

Recall of a previously seen sunset is the recognized, interpreted and retrieved sensory experiences of networks of many of the names listed above in this Section, together with other names such as past, trip, travel, remember, memory and imagine. (A mechanism for recall is proposed below.) Similarly, making a judgment comparing the present sunset with a past sunset would involve names such as compare, different, less, one, change, old, yesterday and now. These processes are outlined in Fig. 3.

\subsection{Specific Illustration of the Model: Recall of Experience and Thought of Names}

To expand on the model laid out above, a simple framework for a complementary mechanism for two higher-order cognitive processes-recall of experience and thinking of names-may be proposed as follows. 


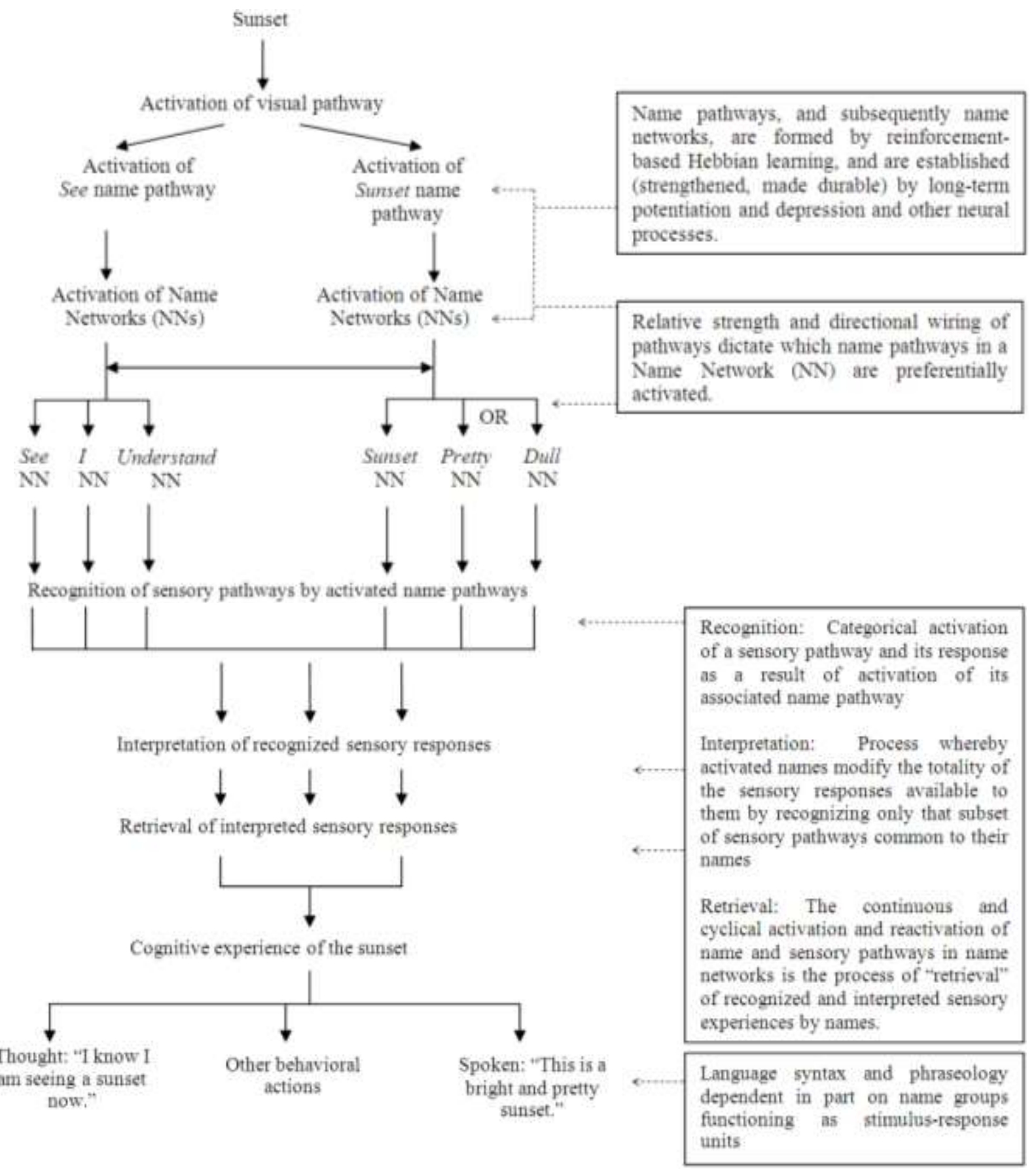

Fig. 3. A name pathway model of higher-order cognition. The visual stimulus of a sunset activates name pathways for see and sunset, previously formed and established as a result of reinforcement-based learning and other processes described in the text. These name pathways activate their Name Networks (NNs), which consist of pathways that are directionally wired and that differ from one another in their relative strength-properties that determine which of two alternate networks is activated. Sensory experiences are recognized, interpreted and retrieved by names, resulting in higher-order cognitive experiences that may be expressed in several ways, such as in thought and speech 


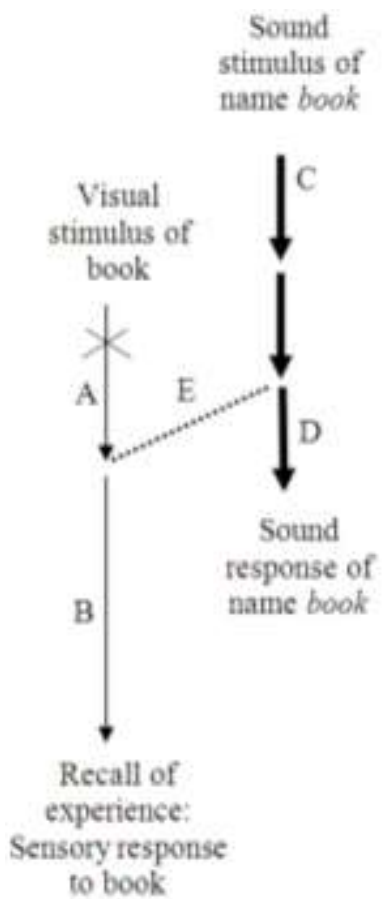

(A)

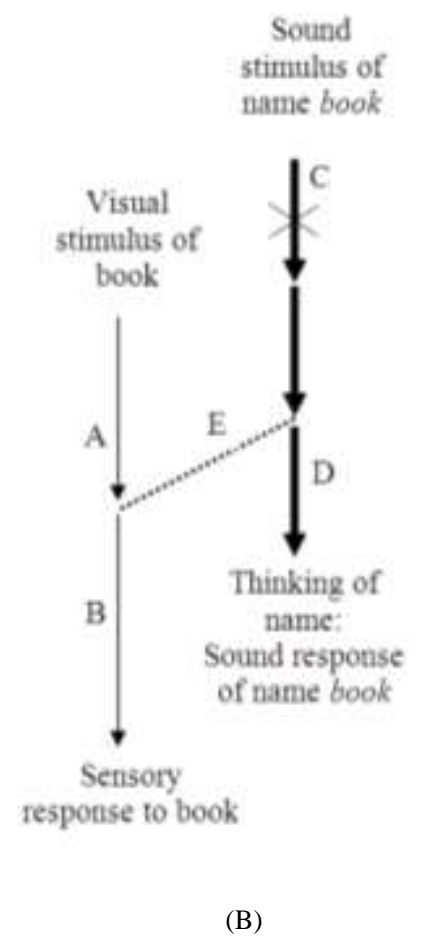

Fig. 4. Mechanism for recall of sensory experience and thinking of names. Pathways in the child are drawn as in Fig. 2. Pathways missing their stimuli are marked with an X. We begin with the condition in which the book visual pathway (pathway A, B) and the book name pathway (C, D) have already been established in the child (with interconnecting pathway E). Figure 4A: Recall of sensory response on hearing a name: The recall of the visual response to an absent book is achieved in pathway $\mathrm{B}$ via activation of C, E, when the book name pathway is activated. Figure 4B: Thinking of a name following a sensory stimulus: The thinking of the name book is achieved in pathway D via activation of $\mathrm{A}, \mathrm{E}$, when the sensory pathway of the book is activated in the absence of hearing the name

A child on hearing the previously learned name of an absent object that he has seen before may behave as if he is responding to its presence, such as reaching for the object. Such "recall of experience" would involve the continual retrieval of the response of the sensory pathway (in the absence its stimulus) via excitation originating from its linked name pathway (Fig. 4A).

Along similar lines, a child when presented with an object that he has earlier learned to name is able to correctly name it again without hearing its name (i.e., in the absence of the name stimulus). This process underlying the "thinking of the name" would involve the continual retrieval of the response of the name pathway (one that is missing its name stimulus) via excitation originating from its linked sensory pathway (Fig. 4B).

Although not discussed further here, a particular advantage of the basic mechanism proposed above is that it can be expanded to account for semantic, non-episodic recall and generalized, abstract thinking.

\section{CONCLUSION}

This elementary theoretical model proposes, for the first time, the existence of a novel class of stimulusresponse pathways called the name pathway, defines its structure and function and describes explicit mechanisms for the emergence of higher-order cognitive functions through name pathway-induced activity in networks of sensory and name pathways. These mechanisms are also among some of the most explicit descriptions of the concepts of the recognition, interpretation and retrieval of sensory information couched in the language of elementary pathway systems. This self-sufficient model is based solely on basic Hebbian learning and neurobiology and involves no more than networks of stimulus-response pathways and pathways that interconnect them. Figure 3, while depicting rudimentary features of such networks, is suggestive of how further layers of detailed and extensive pathways can be added to provide more meaning and 
comprehension (although more complexity) to the model. As discussed earlier, functional activity in such a multitude of networks is largely dependent on the relative strength of the connecting pathways. In this sense, the model approximates those that are built on Hebbian learning and the connectivity of synapses and their strengths (e.g., Hopfield and Tank, 1986; Cowell and French, 2011). Computational testing as applied to such synaptic models may therefore be fruitfully applied to the name pathway model.

Some examples of where the name pathway model may be aligned with current theories and thus may add to our understanding of them may be pointed out here. Name pathways connected to sensory pathways as described here may well function as mirror neurons (Rizzolatti and Craighero, 2004). Thus, regardless of whether I see someone eat a fruit and hear him say "sweet," or I am the one who bites into a fruit and says "sweet," the same visual pathway for fruit, the auditory pathway for "sweet," and the interconnected name pathway networks for fruit and sweet are activated in the observed and the observer. Another example that supports the fundamentals of the name pathway model presented here is the theory of working memory (Repov and Baddeley, 2006), which specifies that verbal information (analogous to the name pathways in the model) and nonverbal information (analogous to the sensory pathways in the model) are held in separate and dissociable (but interconnected, analogous to the connecting pathways in the model) working memory stores.

A major implication of the name pathway model is that by augmenting our repertoire of names with new names (thus creating new name pathways) and by creating new links among names (such as by forming new name groups), we widen our selective as well as ondemand access to specific sensory experiences. One may also speculate that the model may inform the debate on the meaning of cognitive choice and control (Haggard, 2008; Kable and Glimcher, 2009). Our so-called choice of action at each stage of decision-making could be largely a function of the neural experience (as defined previously) of a name pathway and the nature of the stimulus at every such assessment point in a name network. On the other hand, an obvious example where control is minimal is the phenomenon of nighttime dreaming, likely in part to be the result of short bursts of activity in subgroups of name networks that are excited by weak and momentary stimuli from other connected name and sensory pathways. Finally, it could also be speculated that the name pathway model may adequately explain some elements of additional phenomena such as, to provide just one example, psychosomatic control ascribed to meditative thought (Anderson et al., 2008).

\section{REFERENCES}

Anderson, J.W., C. Liu and R.J. Kryscio, 2008. Blood pressure response to transcendental meditation: A meta-analysis. Am. J. Hypertens., 21: 310-316. DOI: 10.1038/ajh.2007.65

Brecht, M. and D. Schmitz, 2008. Rules of plasticity. Science, 319: 39-40. DOI: 10.1126/science.1153231

Casey, B.J., J.N. Giedd and K.M. Thomas, 2000. Structural and functional brain development and its relation to cognitive development. Biol. Psychol., 54: 241-257. DOI: 10.1016/S0301-0511(00)00058-2

Colunga, E. and L.B. Smith, 2005. From the lexicon to expectations about kinds: A role for associative learning. Psychol. Rev., 112: 347-382. DOI: $10.1037 / 0033-295 X .112 .2 .347 \mathrm{a}$

Cowell, R.A. and R.M. French, 2011. Noise and the emergence of rules in category learning: A connectionist model. IEEE Trans. Autonomous Mental Develop., 3: 194-206. DOI: 10.1109/TAMD.2010.2099225

Cummings, A., A.P. Saygin, E. Bates and F. Dick, 2009. Infants' recognition of meaningful verbal and nonverbal sounds. Lang. Learn. Dev., 5: 172-190. DOI: $10.1080 / 15475440902754086$

DeLeon, J., R.F. Gottesman, J.T. Kleinman, M. Newhart and C. Davis et al., 2007. Neural regions essential for distinct cognitive processes underlying picture naming. Brain, 130: 1408-1422. DOI: 10.1093/brain/awm011

Feldman, J. and S. Narayanan, 2004. Embodied meaning in a neural theory of language. Brain Lang., 89: 385392. DOI: 10.1016/S0093-934X(03)00355-9

Frank, M.C., N.D. Goodman and J.B. Tenenbaum, 2009. Using speakers' referential intentions to model early cross-situational word learning. Psychol. Sci., 20: 578-85. DOI: 10.1111/j.1467-9280.2009.02335.x

Garagnani, M., T. Wennekers and F. Pulvermüller, 2008. A neuroanatomically grounded Hebbian-learning model of attention-language interactions in the human brain. Eur. J. Neurosci., 27: 492-513. DOI: 10.1111/j.1460-9568.2008.06015.x

Glotin, H., P. Warnier, F. Dandurand, S. Dufau and B. Lété et al., 2010. An adaptive resonance theory account of the implicit learning of orthographic word forms. J. Physiol. Paris, 104: 19-26.

DOI: 10.1016/j.jphysparis.2009.11.003 
Haggard, P., 2008. Human volition: Towards a neuroscience of will. Nature Rev. Neurosci., 9: 934-46. DOI: $10.1038 / \mathrm{nrn} 2497$

Haider, B., A. Duque, A.R. Hasenstaub and D.A. McCormick, 2006. Neocortical network activity in vivo is generated through a dynamic balance of excitation and inhibition. J. Neurosci., 26: 4535- 4545. DOI: 10.1523/JNEUROSCI.5297-05.2006

Harnad, S., 1990. The symbol grounding problem. Physica D, 42: 335-346. DOI: $10.1016 / 0167-2789(90) 90087-6$

Harris, C.L., 2003. Language and Cognition: Encyclopedia of Cognitive Science. 1st Edn., MacMillan, London.

Hauser, M., 2005. Our chimpanzee mind. Nature, 437: 60-63. DOI: 10.1038/nature03917

Heyes, C., 2012. New thinking: The evolution of human cognition. Phil. Trans. R. Soc. B, 367: 2091-2096. DOI: $10.1098 /$ rstb.2012.0111

Holden, C., 2004. The origin of speech. Science, 303: 1316-1319. DOI: 10.1126/science.303.5662.1316

Hopfield, J.J. and D.W. Tank, 1986. Computing with neural circuits: A model. Science, 233: 625-633. DOI: $10.1126 /$ science. 3755256

Humphreys, G.W., C.J. Price and M.J. Riddoch, 1999. From objects to names: A cognitive neuroscience approach. Psychol. Res., 62: 118-130.

DOI: $10.1007 / \mathrm{s} 004260050046$

Kable, J.W. and P.W. Glimcher, 2009. The neurobiology of decision: Consensus and controversy. Neuron, 63: 733-45. DOI: 10.1016/j.neuron.2009.09.003

Kuhl, P.K., 2000. A new view of language acquisition. Proc. Nat. Acad. Sci. USA, 97: 11850-11857. DOI: 10.1073/pnas.97.22.11850

Mayor, J. and K. Plunkett, 2010. A neurocomputational account of taxonomic responding and fast mapping in early word learning. Psychol. Rev., 117: 1-31. DOI: $10.1037 / \mathrm{a} 0018130$

Newhart, M., L. Ken, J.T. Kleinman, J. Heidler-Gary and A.E. Hillis, 2007. Neural networks essential for naming and word comprehension. Cogn. Behav. Neurol., 20: 25-30.

DOI: $10.1097 /$ WNN.0b013e31802dc4a7

Pinker, S., 1999. Out of the minds of babes. Science, 283: 40-41. DOI: 10.1126/science.283.5398.40

Quiroga, R.Q., L. Reddy, G. Kreiman, C. Koch and I. Fried, 2005. Invariant visual representation by single neurons in the human brain. Nature, 435: 1102-1107. DOI: $10.1038 /$ nature03687
Regier, T., P. Kay and R.S. Cook, 2005. Focal colors are universal after all. Proc. Nat. Acad. Sci. USA, 102: 8386-8391. DOI: 10.1073/pnas.0503281102

Repov, G. and A. Baddeley, 2006. The multi-component model of working memory: Explorations in experimental cognitive psychology. Neurosci.,139: 5-21. DOI: 10.1016/j.neuroscience.2005.12.061

Rizzolatti, G. and L. Craighero, 2004. The mirror-neuron system. Ann. Rev. Neurosci., 27: 169-92. DOI: 10.1146/annurev.neuro.27.070203.144230

Schurger, A., F. Pereira, A. Treisman and J.D. Cohen, 2010. Reproducibility distinguishes conscious from nonconscious neural representations. Science, 327: 97-99. DOI: $10.1126 /$ science. 1180029

Sheridan, S.R., 2005. A theory of marks and mind: The effect of notational systems on hominid brain evolution and child development with an emphasis on exchanges between mothers and children. Med. Hypoth., 64: 417-427.

DOI: 10.1016/j.mehy.2004.09.002

Stroffek, J., E. Kuriscak and P. Marsalek, 2007. Pattern storage in a sparsely coded neural network with cyclic activation. BioSystems, 89: 257-63. DOI: 10.1016/j.biosystems.2006.04.023

The CDC, 2012. The Cambridge declaration on consciousness.

http://fcmconference.org/img/CambridgeDeclaration OnConsciousness.pdf

Thornton, A., N.S. Clayton and U. Grodzinski, 2012. Animal minds: From computation to evolution. Phil. Trans. Royal Soc. London B Biol. Sci., 367: 2670-6. DOI: $10.1098 /$ rstb.2012.0270

Tranel, D., K. Manzel, E. Asp and D. Kemmerer, 2008. Naming dynamic and static actions: Neuropsychological evidence. J. Physiol. Paris, 102: 80-94. DOI: 10.1016/j.jphysparis.2008.03.008

Ulbaek, I, 1998. The Origin of Language and Cognition. In: Approaches to the Evolution of Language: Social and Cognitive Bases, Hurford, J.R., M. StuddertKennedy and C. Knight (Eds.), Cambridge, UK: Cambridge University Press.

Winawer, J., N. Witthoft, M.C. Frank, L. Wu and A.R.Wade et al., 2007. Russian blues reveal effects of language on color discrimination. Proc. Nat. Acad. Sci. USA, 104: 7780-7785. DOI: $10.1073 /$ pnas.0701644104

Wong, K., 2005. The morning of the modern mind. Sci. Am., 292: 86-95.

DOI: $10.1038 /$ scientificamerican0605-86 